\title{
Diferencias de género en la valoración de la intensidad emocional de las expresiones faciales de alegría y tristeza
}

\section{Gender differences in the assessment of the emotional intensity of the facial expressions of happiness and sadness}

\author{
Fernando Gordillo-León'1, Lilia Mestas-Hernández², Miguel A. Pérez-Nieto' y José M. Arana-Martínez ${ }^{3}$ \\ 1 Universidad Camilo José Cela, España. ${ }^{2}$ Facultad de Estudios Superiores Zaragoza, México. \\ ${ }^{3}$ Universidad de Salamanca, España
}

\begin{abstract}
Resumen
Las diferencias de género en el reconocimiento de las emociones podrían deberse a una mayor sensibilidad de las mujeres a las señales sutiles de las expresiones faciales. El objetivo de esta investigación consistió en analizar las diferencias de género en la valoración de los cambios en la intensidad emocional de las expresiones faciales de alegría $y$ tristeza. La muestra estuvo formada por 222 participantes (77.5\% mujeres), con edades comprendidas entre los $18 \mathrm{y}$ 30 años. Se utilizaron expresiones faciales ambiguas con diferentes porcentajes de alegría y tristeza (ambigüedad alta: $50 \%-50 \%$, ambigüedad media: $25 \%-75 \%$, ambigüedad baja: $0 \%-100 \%$ ). Los participantes valoraron en qué grado una expresión ambigua reflejaba mayor intensidad emocional (alegría o tristeza) que una expresión neutra. Las mujeres percibieron mayor intensidad emocional en las expresiones de alegría con ambigüedad media ( $25 \%$ tristeza - $75 \%$ alegría) en comparación con los hombres. Además, solo las mujeres percibieron mayor intensidad afectiva en las expresiones de alegría (25\% tristeza - $75 \%$ alegría) respecto a las de tristeza (75\% tristeza - $25 \%$ alegría) con ambigüedad media. Se discuten los resultados a partir de la teoría del rol social, y se analizan sus implicaciones dentro del complejo contexto social en el que interaccionan los seres humanos.
\end{abstract}

Palabras clave: Alegría, intensidad emocional, sensibilidad, tristeza.

\begin{abstract}
Differences between men and women in the recognition of emotions could be due to the greater sensitivity of women to the subtle signals of emotional facial expressions. The objective of this research was to analyze gender differences in the assessment of changes in the emotional intensity of facial expressions of happiness and sadness. The sample comprised 222 participants (77.5\% women) aged between 18 and 30 years. Ambiguous facial expressions were presented with different percentages of happiness and sadness (high ambiguity: $50 \%-50 \%$; medium ambiguity: $25 \%-75 \%$; low ambiguity: $0 \%-100 \%$ ). The participants rated the degree to which an ambiguous expression reflected greater emotional intensity (happiness or sadness) than a neutral expression. Under conditions of medium ambiguity (25\% sadness - $75 \%$ happiness), greater emotional intensity in expressions of happiness was perceived by women than men. Also under medium ambiguity, women perceived greater affective intensity in expressions of happiness (25\% sadness - $75 \%$ happiness) than in expressions of sadness $(75 \%$ sadness - $25 \%$ happiness). The results are discussed from the perspective of social role theory, and their implications are analyzed within the complex social context in which human beings interact.
\end{abstract}

Keywords: Happiness, emotional intensity, sensitivity, sadness.

Cómo citar este artículo: Gordillo-León, F., Mestas-Hernández, L., Pérez-Nieto, M.A., Arana-Martínez, J.M. (2021). Diferencias de género en la valoración de la intensidad emocional de las expresiones faciales de alegría y tristeza. Escritos de Psicología - Psychological Writings, 14(1), 1-10. https://doi.org/10.24310/espsiescpsi.v14i1.12675 


\section{Introducción}

Las emociones desempeñan una importante función adaptativa preparando al organismo para reaccionar ante cambios relevantes en el contexto y transmitiendo la información de manera rápida y eficaz. Hay una significativa variabilidad interpersonal en el procesamiento emocional, siendo las diferencias de género y de la edad las que mayor atención han atraído en los últimos años (e.g., Abbruzzese et al., 2019). Se ha considerado que las mujeres son más emocionales que los hombres, en concreto respecto a las emociones negativas (Gard \& Kring, 2007) las cuales son experimentadas con una mayor intensidad y frecuencia (Bradley et al., 2001; Fernández et al., 2012). Sin embargo, los hombres manifiestan reacciones emocionales más intensas ante estímulos amenazantes (Kret \& De Gelder, 2012) y eróticos (Karama et al., 2002). Se ha encontrado en las mujeres, respecto a los hombres, una mayor facilidad en el uso del lenguaje emocional (Fugate et al., 2009), y una mayor tendencia a sonreír y llorar (Fischer \& Lafrance, 2015; LaFrance et al., 2003). También se ha encontrado diferencias de género en la forma en la que se expresan las emociones. Mientras que las mujeres lo hacen con más frecuencia verbalmente y a través del rostro, los hombres se expresan más a través del comportamiento (Kret \& De Gelder, 2012), experimentando y expresando ira con mayor frecuencia (Biaggio, 1980, 1989; Doyle \& Biaggio, 1981). Estas diferencias podrían estar mediadas por los roles sociales, las limitaciones situacionales y la intensidad emocional (Fischer \& Lafrance, 2015). Además, la supuesta mayor emocionalidad de las mujeres respecto a los hombres, no siempre se cumple en las culturas occidentales, lo que podría ser una evidencia del efecto de las normas sociales sobre la forma en la que se expresan las emociones (Gong et al., 2018).

Respeto al procesamiento de la expresión emocional, las mujeres muestran una mayor eficacia (Hall, 1978; McClure, 2000; Thompson \& Voyer, 2014), y rapidez (Kret \& De Gelder, 2012; Saylik et al., 2018) en el reconocimiento de las emociones a través del rostro, excepto en la expresión de ira (Di Tella et al., 2020). Estas diferencias también se mantienen cuando se utilizan evaluaciones subjetivas como indicadores de la expresividad emocional, encontrándose que las mujeres valoran con mayor intensidad la emociones con independencia de la valencia (Barrett et al., 1998; Grossman \& Wood, 1993; Vrana \& Rollock, 2002). Sin embargo, otros estudios no han encontrado diferencias utilizando estímulos como caras (Wild et al., 2001) o películas (Carvalho et al., 2012). En general, hay cierto consenso respecto a una mayor eficacia de las mujeres en el reconocimiento facial de las emociones, aunque estas diferencias se vuelven menos claras cuando se analizan los diferentes tipos de emociones por separado. Se ha planteado que estas diferencias estarían determinadas por una mayor sensibilidad de las mujeres en la detección de cambios sutiles en la expresión facial cuando las emociones son menos intensas (Hipótesis de la sensibilidad emocional, Hoffmann et al., 2010; Montagne \& Kessels, 2005). Esta mayor sensibilidad de las mujeres podría estar determinada por los estereotipos de género (Niedenthal et al., 2006), que establecerían una función social diferente a hombres y mujeres (teoría del rol social, Eagly et al., 2000). Por un lado, las mujeres se involucrarían en mayor grado en el cuidado de los niños, y el hombre en la provisión de los recursos de subsistencia (Spence \& Helmreich, 1978). En las situaciones de cuidado se espera un intercambio social positivo y de cohesión grupal, por lo que las expresiones de alegría y tristeza serían más frecuentemente utilizadas por la mujer. Por otro lado, los hombres se involucran en contextos de competencia que con frecuencia derivan en conflictos, y donde la expresión de ira tendría una mayor predominancia. Bajo esta perspectiva, hombres y mujeres evidenciarían un uso instrumental de las emociones para favorecer su adaptación al contexto social que podría estar determinando la frecuencia con la que expresan determinadas emociones, y la habilidad que han adquirido para reconocerlas. Sin embargo, recientes investigaciones no encuentran que el género determine diferencias claras en la valoración de la intensidad emocional. Si bien, en términos generales, informan de un mejor rendimiento de las mujeres en el reconocimiento de las expresiones emocionales (Wingenbach et al., 2018), no quedaría claro que se mantenga para todo tipo de emociones y situaciones (Connolly et al., 2019).

Respecto a las emociones de alegría y tristeza, algunas evidencias con técnicas de resonancia magnética funcional apoyan el supuesto de que en hombres y mujeres se activan áreas cerebrales diferentes durante el reconocimiento de este tipo de emociones (Lee et al., 2002; Kinoshita et al., 2012). Sin embargo, algunos estudios no han encontrado diferencias de género en la identificación de las emociones de alegría y tristeza (Rahman et al., 2004), tampoco en los tiempos de reacción (Grimshaw et al., 2004), ni en tareas de detección (ira y alegría) (Sawada et al., 2014). Tampoco a través de vídeos con expresiones faciales en movimiento (Lyusin \& Ovsyannikova, 2016). Otros estudios han encontrado una superioridad de las mujeres en el reconocimiento de la tristeza, pero no de la alegría (Connolly el tal., 2019). La explicación de la falta de congruencia entre los estudios podría encontrarse en las características de la muestra y la metodología utilizada. Por ejemplo, el uso de expresiones faciales 
prototípicas en los experimentos podría generar un efecto techo (e.g., Hampson et al., 2006; Hoffmann et al., 2010), impidiendo mostrar las diferencias de género entre los participantes (Connolly \& Lefevre, 2019). Es decir, con expresiones prototípicas no se evidenciaría la mayor sensibilidad emocional de las mujeres en la detección de las señales sutiles de las expresiones emocionales porque las expresiones faciales utilizadas serían prototipos emocionales fácilmente identificables. En este sentido, una reciente investigación, utilizando una amplia muestra y estímulos faciales con diferentes niveles de intensidad emocional, no encontró diferencias de género que pudieran validar la hipótesis de la mayor sensibilidad de las mujeres a los detalles sutiles (Fischer et al., 2018). Sin embargo, en este trabajo se valoraron las expresiones faciales respecto a la intensidad mostrada, pero no se midió la capacidad de los participantes para determinar con precisión las diferencias en intensidad entre dos expresiones faciales. Quizá esta sea la clave para contrastar adecuadamente la hipótesis de la sensibilidad de género, en tanto que el reconocimiento de la expresión emocional viene determinado en alto grado por el contexto en el que se produce la percepción de la expresión facial (Calbi et al., 2019).

Atendiendo a todos estos aspectos, el objetivo de la presente investigación consistió en analizar las diferencias de género en la valoración de la intensidad emocional de expresiones faciales ambiguas de alegría y tristeza. Se utilizaron expresiones faciales preparadas con morphing para evidenciar en mayor grado las posibles diferencias de género (Hassin et al., 2013), a través de la ambigüedad de los estímulos utilizados. Al mismo tiempo se utilizó una metodología de comparación y valoración de la intensidad emocional percibida, en la que los participantes tuvieron que valorar una expresión facial ambigua, comparándola con una expresión facial neutra. Por lo tanto, no se hablaría en términos de reconocimiento sino de valoración de la intensidad emocional percibida en un rostro. Si las diferencias de género derivan de la diferente sensibilidad emocional entre hombres y mujeres (Hipótesis de la sensibilidad emocional, Hoffmann et al., 2010; Montagne \& Kessels, 2005), estas diferencias se mostrarán con mayor facilidad cuando se valoran los cambios en la intensidad de una emoción, porque supone una perspectiva dimensional (niveles de intensidad) con un mayor rango de valores que la perspectiva categorial (tipo de emoción). Esta metodología se adecua mejor a las situaciones reales, donde las valoraciones que se realizan de las emociones provienen en muchas ocasiones de procesos de comparación con estados anteriores o neutros, dando lugar a cambios sutiles y progresivos. Por lo tanto, se prevé que las mujeres valoren con mayor intensidad los cambios en las expresiones de alegría y tristeza que los hombres, siendo estas diferencias mayores con niveles de ambigüedad media $(25 \% / 75 \%)$ donde los cambios en la intensidad emocional serían más sutiles.

\section{Participantes}

\section{Método}

En el experimento participaron 222 estudiantes universitarios de la Facultad de Estudios Superiores Zaragoza (México), con edades comprendidas entre los 18 y los 30 años $(77.5 \%$ Mujeres; $M=22.10$, $D T=3.05)$. Se realizó una comparación de medias ( $t$ de Student) entre hombres y mujeres en la variable edad, sin encontrarse diferencias significativas, $t(220)=1.48, p=.14$. Todos los participantes lo hicieron de manera voluntaria tras leer y aceptar el consentimiento informado.

\section{Instrumentos}

Se utilizaron seis expresiones faciales prototípicas de dos modelos (hombre y mujer): dos neutras, dos de alegría y dos de tristeza, obtenidas de la base de datos NimStim Face Stimulus (Tottenham et al., 2009), para elaborar expresiones faciales ambiguas mediante FantaMorph software (Abrosoft, 2010). Se prepararon dos expresiones (hombre y mujer) con ambigüedad alta (50\% alegría- $50 \%$ tristeza) dos expresiones de alegría (hombre y mujer) con ambigüedad media ( $25 \%$ tristeza $-75 \%$ alegría), y dos de tristeza (hombre y mujer) con ambigüedad media (75\% tristeza $-25 \%$ alegría). Las expresiones con ambigüedad baja se correspondían con las expresiones prototípicas (100\% alegría, $100 \%$ tristeza) (véase Figura 1). Con este tipo de estímulos se espera que la sensibilidad de la tarea mejore, al incluir matices emocionales sutiles en la expresión facial (Wieser \& Brosch, 2012; Matsumoto \& Hwang, 2010).

\section{Procedimiento}

El experimento se aplicó on-line a través de Google Form. Se utilizó el muestro bola de nieve en estudiantes de los primeros cursos de Psicología de la Facultad de Estudios Superiores Zaragoza (México). El experimento constaba de una primera fase en la que se les facilitó a los participantes el consentimiento informado junto con una dirección de consulta. Posteriormente se les pidió que indicarán el género, la edad, el estado civil y la nacionalidad. Por último, realizaron la tarea de valoración de la intensidad emocional de expresiones faciales. En cada ensayo, de un total de doce presentados alea- 
toriamente, les aparecían en la pantalla dos expresiones faciales. La de la izquierda siempre era una expresión neutra, mientras que la de la derecha variaba en los porcentajes que tenía de cada emoción (50\% alegría - $50 \%$ tristeza; $75 \%$ alegría - $25 \%$ tristeza; $25 \%$ alegría - $75 \%$ tristeza; $0 \%$ tristeza - $100 \%$ alegría; $100 \%$ tristeza - 0\% alegría). En total realizaron doce tareas de comparación (Figura 1), seis con expresiones de hombre y seis con expresiones de mujer en las que se tenía que valorar de 1 (muy poco) a 10 (mucho) el cambio en la expresión de la imagen de la derecha respecto a la expresión de neutralidad de la imagen de la izquierda (¿en qué grado considera que la expresión de la derecha está más alegre/triste que la expresión de la izquierda?).

\section{Figura 1}

Procedimiento experimental con los doce ensayos de valoración que se presentaron en el experimento.

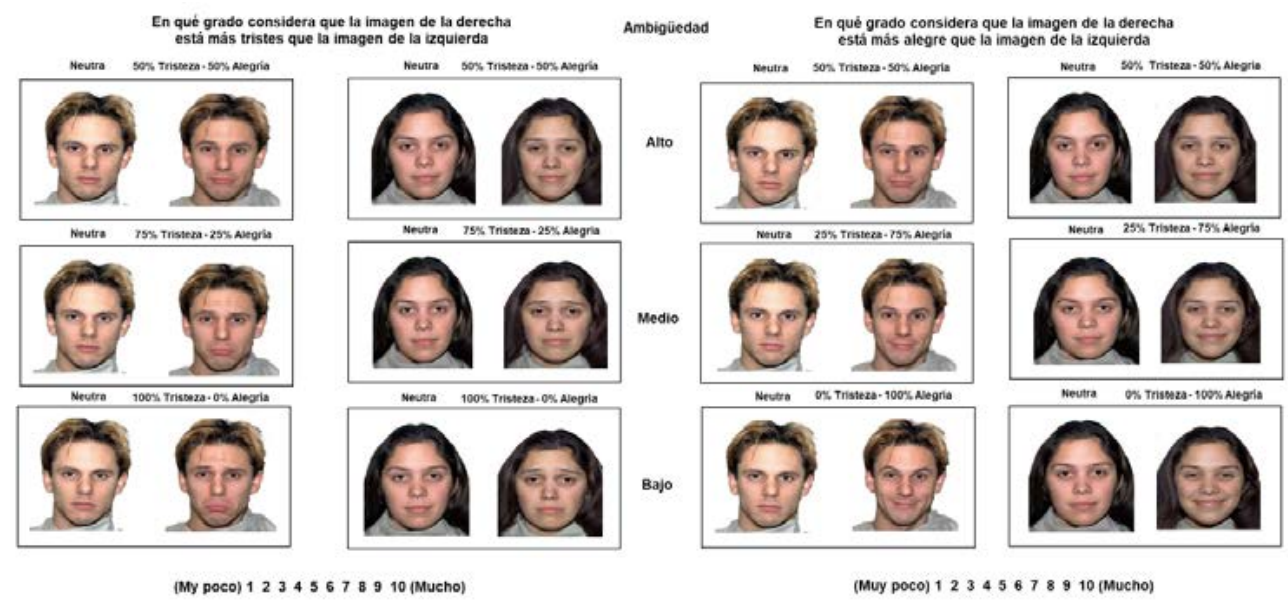

Análisis de datos

Se realizó un ANOVA mixto $2 \times 2 \times 3$, con el Género de los participantes (masculino, femenino), como variable intersujetos, y las variables Emoción (tristeza, alegría), y Ambigüedad (alta: $50 \%$ - 50\%; media: $25 \%-75 \%$, baja: $0 \%-100 \%$ ) como variables intrasujetos. La variable dependiente fue la valoración de la intensidad emocional, con un rango del 1 al 10.

\section{Resultados}

En la Tabla 1 se exponen los estadísticos descriptivos de las condiciones experimentales. En la Tabla 2 se muestran los resultados del ANOVA $2 \times 2 \times 3$. Los efectos principales de los factores Emoción y Ambigüedad resultaron estadísticamente significativos, pero no lo fue el del factor Género. Por otro lado, no se encontró efecto significativo de la interacción entre Género y Ambigüedad, ni entre Género y Emoción, pero sí de la interacción entre Emoción y Ambigüedad, y entre Género, Emoción y Ambigüedad.

Tabla 1

Descriptivos (media y desviación típica) de la valoración de la intensidad emocional según los factores Género, Emoción y Ambigüedad.

\begin{tabular}{lllll} 
& \multicolumn{3}{c}{ Hombres } & Mujeres \\
\cline { 2 - 5 } Ambigüedad baja & Alegría & Tristeza & Alegría & Tristeza \\
Ambigüedad media & $6.89(.29)$ & $5.61(.29)$ & $7.43(.15)$ & $5.46(.16)$ \\
Ambigüedad alta & $5.32(.27)$ & $4.87(.28)$ & $5.95(.14)$ & $4.88(.15)$ \\
\hline
\end{tabular}

Tabla 2

Resultados del ANOVA Género x Emoción x Ambigüedad.

\begin{tabular}{lccc}
\hline Fuentes de variación & $F$ & $p$ & $\eta^{2}$ \\
\hline Género & .94 & .33 & .00 \\
Emoción & 46.53 & $<.001$ & .18 \\
Ambigüedad & 228.01 & $<.001$ & .68 \\
Género*Emoción & 1.67 & .20 & .01 \\
Género*Ambigüedad & .53 & .59 & .01 \\
Emoción*Ambigüedad & 22.79 & $<.001$ & .17 \\
Género*Emoción*Ambigüedad & 3.58 & .03 & .03 \\
\hline
\end{tabular}


El análisis de los efectos simples (método Bonferoni) de la interacción entre Emoción y Ambigüedad, evidenció que las diferencias entre las emociones de tristeza $\left(M_{i}=5.54, E T=.16\right)$ y alegría $\left(M_{i}=\right.$ 7.16, $E T=.16)$ se dieron en los estímulos con niveles de ambigüedad baja $\left(M_{(i-i)}=-1.62, p<.0001, E T\right.$ $=.16)$. En el mismo sentido se observaron diferencias entre tristeza $\left(M_{i}=4.87, E T=.16\right)$ y alegría $\left(M_{i}\right.$ $=5.64, E T=.15)$ en los estímulos con ambigüedad media $\left(M_{(i-\mathrm{j})}=-.77, p<.0001, E T=.16\right)$ (Figura 2).

Figura 2

Valores promedio de la valoración de la intensidad emocional en la interacción entre Emoción y Ambigüedad. * $p<.0001$. Las barras de error representan el error típico.

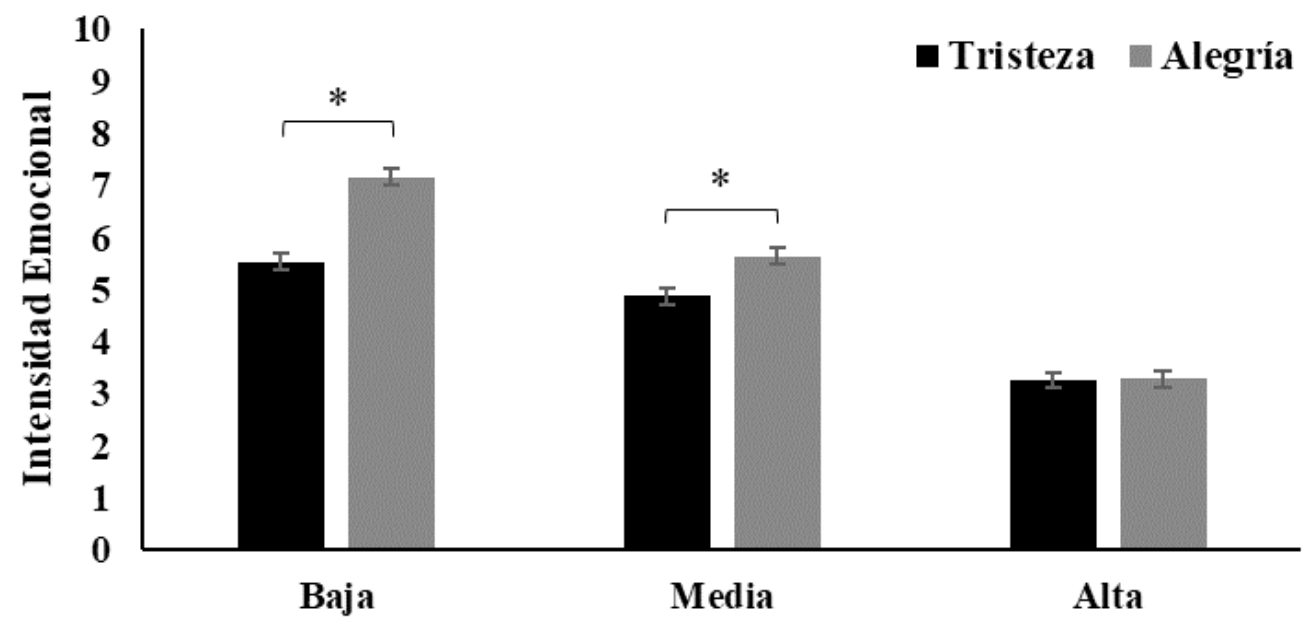

\section{Ambigüedad}

Respecto a la interacción entre Género, Emoción y Ambigüedad, el análisis de los efectos simples mostró que las diferencias entre las emociones de tristeza y alegría se dieron con niveles de ambigüedad baja, tanto en hombres $\left(M_{(i-\mathrm{j})}=-1.28, p<.0001, E T=.29\right)$, como en mujeres $\left(M_{(i-\mathrm{i})}=-1.98\right.$, $p<.0001, E T=.16)$. También con niveles de ambigüedad media, pero solo en mujeres $\left(M_{(i-i)}=-1.08, p\right.$ $<.0001, E T=.15)$. Además, se mostraron diferencias entre hombres y mujeres con niveles de ambigüedad media en la valoración de la expresión de alegría $\left(M_{(i-j)}=-.63, p=.038, E T=.30\right)$ (Figura 3).

Figura 3

Valores promedio de la valoración de la intensidad emocional en la interacción entre Género, Emoción y Ambigüedad. ${ }^{*} p<.05^{* *} p<.0001$. Las barras de error representan el error típico.

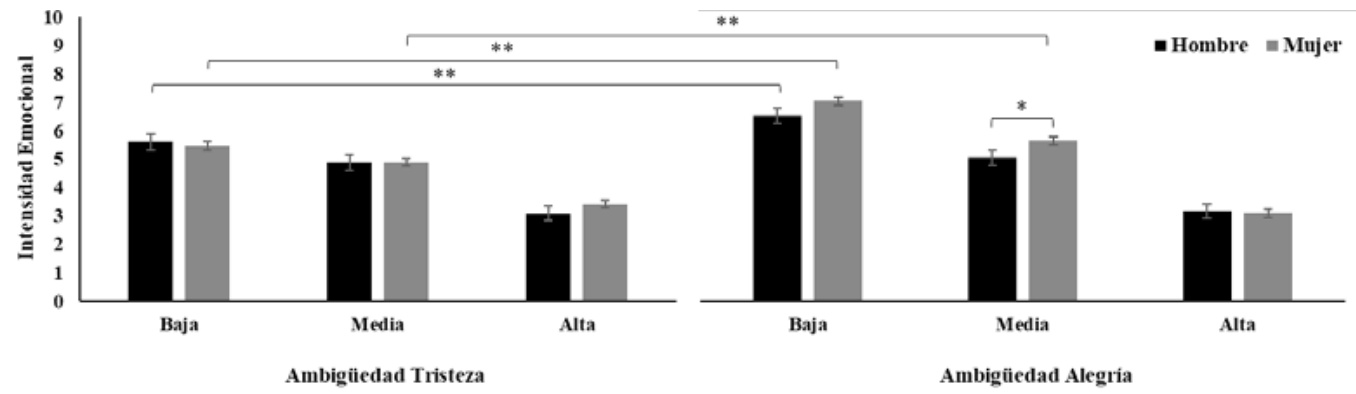

\section{Discusión}

El objetivo de la presente investigación fue el de analizar las diferencias entre hombres y mujeres en la valoración de la intensidad emocional percibida en expresiones ambiguas de alegría y tristeza. Los resultados obtenidos permiten aceptar la hipótesis experimental planteada, dado las mujeres valoraron con mayor intensidad las expresiones de alegría en la condición de ambigüedad media en comparación con los hombres. Además, las diferencias entre las expresiones de alegría y tristeza se muestran de manera significativa en las mujeres, cuando la ambigüedad fue baja y media, mientras que en los hombres solo cuando la ambigüedad fue baja. Estos resultados podrían explicarse por la mayor sensibilidad de las mujeres a la hora de detectar cambios sutiles en las señales emocionales (Hoffmann et al., 2010; Montagne \& Kessels, 2005). 
La mayor sensibilidad de las mujeres podría estar determinada por la cultura, el rol de género y las normas sociales (Kret \& De Gelder, 2012). En concreto, la teoría del rol social (Eagly et al., 2000) propone una explicación a partir del diferente rol que los hombres y las mujeres han tenido a lo largo de su historia evolutiva, y que habría determinado los estereotipos de género actuales. Bajo esta perspectiva, la mujer habría adquirido un uso más frecuente e instrumental de la expresión de alegría por su mayor compromiso con la cohesión del grupo. En consecuencia, también podría haber adquirido una mayor capacidad para detectar dicha expresión facial. Los resultados de algunos estudios parecen apoyar esta teoría, en tanto que han evidenciado que las mujeres manifiestan una mayor facilidad a la hora de detectar la sonrisa genuina (Hall, 1984), tienden a utilizarla con mayor frecuencia y la asocian en mayor grado con la honestidad (Krys et al., 2015). Estos datos apuntan a diferencias instrumentales en la utilización de la sonrisa entre hombres y mujeres con una clara implicación en el ámbito social. Por otro lado, se ha documentado que los hombres reaccionan con una mayor activación fisiológica que las mujeres cuando observan la expresión facial de ira en un hombre (Mazurski et al., 1996; Fischer et al., 2004). Bajo la perspectiva de la teoría del rol social esto sería debido a que en el hombre la reproducción se asocia a la rivalidad directa con otros competidores, lo que es congruente con el hecho de que en la mayoría de las culturas los hombres son los que con mayor frecuencia comente delitos violentos (Daly \& Wilson, 1988).

Si bien algunos estudios de meta-análisis evidencian que las mujeres tienen una mayor capacidad que los hombres a la hora de reconocer las emociones a través del rostro (Hall, 1978; Hall et al., 2000), estas diferencias tienen un tamaño del efecto pequeño (Hyde, 2014), que podría explicarse por la utilización, en la mayoría de los estudios analizados, de expresiones faciales prototípicas que tenderían a igualar en la respuesta a ambos géneros. Esto es congruente con aquellos estudios que han encontrado que las diferencias son mayores entre hombres y mujeres cuando se reduce el tiempo de exposición de las expresiones faciales (menos de un segundo) (Hall \& Matsumoto, 2004; Hoffmann et al., 2010); aspecto que se ha constatado respecto a las expresiones de alegría utilizando el paradigma de priming (Donges et al., 2012). Otras investigaciones utilizando priming pero con presentaciones visibles de las expresiones faciales (400 ms) (Gohier et al., 2011), parecen evidenciar que las mujeres son más sensibles a expresiones de amenaza que los hombres cuando se produce a un nivel de consciencia mayor. Por lo tanto, el nivel de consciencia a la hora de reconocer una expresión emocional podría ser una variable relevante para comprender las diferencias de género.

En el complejo contexto social actual, la expresión facial de las emociones se ha convertido en un sutil sistema de transmisión de información. Su valor ya no radica en reconocer una emoción prototípica en el rostro de otras personas, sino en la capacidad de interpretar los sutiles cambios en las señales emocionales del rostro durante los procesos de interacción social, que estarían determinando en gran parte la deriva de las relaciones entre las personas en diferentes ámbitos. Por ejemplo, la expresión sutil de ira conlleva algunos beneficios en la negociación (Van Kleef \& De Dreu, 2010). Otro ejemplo de la importancia de la expresión sutil de las emociones y su repercusión en el contexto social, son los datos encontrado respecto a las dificultades que presentan las personas con depresión a la hora de detectar cambios en la intensidad de las expresiones de alegría (Yoon et al., 2009). No detectar estos cambios les impediría percibir matices en la comunicación interpersonal que enriquecen su contenido y por lo tanto les aislaría de su entorno social.

Estas diferencias de género también se evidencian en el uso tecnológico, donde las mujeres reportan utilizar con mayor frecuencia emojis, manifestando hacia ellos una actitud más positiva (Prada et al., 2018). Sin embargo, no se aprecian diferencias significativas en la interpretación que los hombres y mujeres realizan de los emoticonos en redes sociales (e.g., Facebook; Herring \& Dainas, 2020). No obstante, al igual que en los estudios que han utilizado expresiones faciales reales, en este ámbito los emojis analizados expresan emociones prototípicas que podrían estar igualando a hombres y mujeres en su rendimiento.

Los resultados encontrados en la presente investigación advierten de la importancia de analizar las diferencias en la percepción de los cambios en la intensidad de las emociones entre hombres y mujeres, más allá de la capacidad en términos absolutos de reconocer los diferentes tipos básicos de emoción. En este sentido, y a partir de los resultados encontrados en la presente investigación, se podría afirmar que las mujeres perciben con mayor intensidad los cambios de las expresiones de alegría. Estas diferencias podrían dar lugar a interpretaciones diferentes de los gestos, en diferentes ámbitos como el de las relaciones personales, la política y la educación, donde la comunicación no verbal enriquece el discurso y participa en los procesos de toma de decisiones. Además, estos resultados son congruentes con las diferencias entre hombres y mujeres en capacidades que necesitan de una adecuada precisión en el reconocimiento de las emociones. En este sentido, algunos estudios han encontrado que las mujeres son mejores en la comprensión de las emociones de los demás (Barret 
\& Bliss-Moreau, 2009; Shields, 2002), y puntúan más alto que los hombres en empatía o inteligencia emocional (Davis, 1980; Barrett et al., 2000; Ciarrochi et al., 2005; Schutte et al., 1998).

Se podría concluir con los siguientes puntos : a) El tipo de estímulo, las condiciones de presentación, y el tipo de paradigma utilizado podrían estar determinando las inconsistencias respecto a las diferencias de género en el reconocimiento de las expresiones faciales de la emoción; b) los resultados obtenidos son congruentes con aquellas investigaciones que informan de un mayor efecto de las diferencias de género cuando las expresiones faciales no son prototípicas; y c) la valoración del cambio en la intensidad emocional resulta un método adecuado para el estudio de las diferencias de género, porque permite evidenciar la mayor sensibilidad de las mujeres a los cambios de intensidad afectiva, en concreto en lo referido a las expresiones faciales de alegría.

Las limitaciones del trabajo están referidas al diferente porcentaje de la muestra, siendo mayor el de mujeres que el de hombres, así como en el formato de aplicación on-line. También debería tenerse en cuenta que al ser una muestra de universitarios la generalización de los resultados se podría ver comprometida. Por otro lado, sería conveniente incrementar el número de modelos faciales utilizados, para evitar sesgos derivados de las peculiaridades de cada uno de ellos (aspectos físicos, atractivo, etc.). Futuras investigaciones deberían analizar, aplicando la misma metodología, las diferencias entre hombres y mujeres en el resto de las expresiones emocionales (ira, asco, miedo, sorpresa), así como incluir en el estudio muestras de poblaciones clínicas, cuyo trastorno curse con déficit en el reconocimiento de las expresiones faciales emocionales.

\section{Agradecimientos}

Este trabajo ha sido financiado por la Universidad Camilo José Cela (VI Convocatoria de Ayudas a la Investigación UCJC).

\section{Referencias}

1. Abbruzzese, L., Magnani, N., Robertson, I. H., \& Mancuso, M. (2019). Age and gender differences in emotion recognition. Frontiers in Psychology, 10, 2371. https://doi.org/10.3389/ fpsyg.2019.02371

2. Abrosoft (2010). FantaMorph (Version 5.0) [Computer software].

3. Barrett, L. F., \& Bliss-Moreau, E. (2009). She's emotional. He's having a bad day: Attributional explanations for emotion stereotypes. Emotion, 9, 649-658. https://doi.org/10.1037/a0016821

4. Barrett, L. F., Lane, R. D., Sechrest, L., \& Schwartz, G. E. (2000). Sex differences in emotional awareness. Personality and Social Psychology Bulletin, 26, 1027-1035. https://doi. org/10.1177/01461672002611001

5. Barrett, L. F., Robin, L., Pietromonaco, P. R., \& Eyssell, K. M. (1998). Are women the "moreemotional" sex? Evidence from emotional experiences in social context. Cognition \& Emotion, 12, 555-578. 15. https://doi.org/10.1080/026999398379565

6. Biaggio, M. K. (1980). Assessment of anger arousal. Journal of Personality Assessment, 44(3), 289-298. https://doi.org/10.1207/s15327752jpa4403 12

7. Biaggio, M. K. (1989). Sex differences in behavioral reactions to provocation of anger. Psychological Reports, 64(1), 23-26. https://doi.org/10.2466/pr0.1989.64.1.23

8. Bradley, M. M., Codispoti, M., Sabatinelli, D., \& Lang, P. J. (2001). Emotion and motivation II: sex differences in picture processing. Emotion, 1(3), 300-319. https://doi.org/10.1037/15283542.1.3.300

9. Calbi, M., Siri, F., Heimann, K. Barratt, D., Gallese, V., Kolesnikov, A., \& Umiltà, M. A. (2019). How context influences the interpretation of facial expressions: a source localization high-density EEG study on the "Kuleshov effect". Scientific Report, 9. https://doi.org/10.1038/s41598-018-37786-y

10. Carvalho, S., Leite, J., Galdo-Álvarez, S., \& Gonçalves, Ó. F. (2012). The emotional movie database (EMDB):A self report and psychophysiological study. Applied Psychophysiology and Biofeedback, 37(4), 279-294. https://doi.org/10.1007/s10484-012-9201-6

11. Ciarrochi J, Hynes K, \& Crittenden N. (2005). Can men do better if they try harder: Sex and motivational effects on emotional awareness. Cognition \& Emotion, 19(1), 133-141. https://doi. org/10.1080/02699930441000102

12. Connolly, H. L., Lefevre, C. E., Young, A. W., \& Lewis, G. J. (2019). Sex differences in emotion recognition: Evidence for a small overall female superiority on facial disgust. Emotion, 19(3), 455-464. https://doi.org/10.1037/emo0000446

13. Daly, M., \& Wilson, M. (1988). Evolutionary social psychology and family homicide. Science, 242(4878), 519-524. https://doi.org/10.1126/science.3175672 
14. Davis, M. H. (1980). A multidimensional approach to individual differences in empathy. Journal of Personality and Social Psychology 10(85).

15. Di Tella, M., Miti, F., Ardito, R., \& Adenzato, M. (2020). Social cognition and sex: Are men and women really different? Personality and Individual Differences, 162(1), 110045. https://doi. org/10.1016/j.paid.2020.110045

16. Donges, U-S., Kersting, A., \& Suslow. T (2012) Women's greater ability to perceive happy facial emotion automatically: gender differences in affective priming. PLOS ONE 7(7), e41745. https:// doi.org/10.1371/journal.pone.0041745

17. Doyle, M.A., \& Biaggio, M. K. (1981). Expression of anger as a function of assertiveness and sex. Journal of Clinical Psychology, 37(1), 154-157. https://doi.org/10.1002/1097-4679(198101) 37:1<154::aid-jclp2270370130>3.0.c0:2-I

18. Eagly, A. H., Wood, W., \& Diekman, A. B. (2000). Social role theory of sex differences and similarities: A current appraisal. In T. Eckes \& H. M. Trautner (Eds.), The developmental social psychology of gender (pp. 123-174). Erlbaum.

19. Fernández, C., Pascual, J. C., Soler, J., Elices, M., Portella, M. J., \& Fernández-Abascal, E. (2012). Physiological responses induced by emotion-eliciting films. Applied Psychophysiology and Biofeedback volume, 37(2),73-79. https://doi.org/10.1007/s10484-012-9180-7

20. Fischer, A. H., \& Lafrance, M. (2015). What drives the smile and the tear: Why women are more emotionally expressive than men. Emotion Review, 7(1), 22-29. https://doi. org/10.1177/1754073914544406

21. Fischer, A. H., Kret, M. E., \& Broekens, J (2018). Gender differences in emotion perception and selfreported emotional intelligence: A test of the emotion sensitivity hypothesis. PLOS ONE, 13(1), e0190712. https://doi.org/10.1371/journal.pone.0190712

22. Fischer, A. H., Rodriguez-Mosquera, P. M., van Vianen, A. E., \& Manstead, A. S. (2004). Gender and culture differences in emotion. Emotion, 4(1), 87-94. https://doi.org/10.1037/1528-3542.4.1.87

23. Fugate, J. M. B., Gouzoules, H., \& Barrett, L. F. (2009). Separating production from perception: Perceiver-based explanations for sex differences in emotion. Behavioural and Brain Sciences, 5, 394-395. https://doi.org/10.1017/S0140525X09990203

24. Gard, M. G., \& Kring, A. M. (2007). Sex differences in the time course of emotion. Emotion, 7(2), 429-437. https://doi.org/10.1037/1528-3542.7.2.429

25. Gohier, B., Senior, C., Brittain, P. J., Lounes, N., \& El-Hage, W. (2011). Gender differences in the sensitivity to negative stimuli: Cross-modal affective priming study. European Psychiatry, 28(2), 74-80. https://doi.org/10.1016/j.eurpsy.2011.06.007.

26. Gong, X., Wong, N., \& Wang, D. (2018). Are Gender Differences in Emotion Culturally Universal? Comparison of Emotional Intensity Between Chinese and German Samples. Journal of Cross-Cultural Psychology, 49(6), 993-1005. https://doi.org/10.1177/0022022118768434

27. Grimshaw, G. M., Bulman-Fleming, M. B., \& Ngo, C. (2004). A signal-detection analysis of sex differences in the perception of emotional faces. Brain and Cognition, 54(3), 248-250. https://doi. org/10.1016/j.bandc.2004.02.029

28. Grossman, M., \& Wood, W. (1993). Sex differences in intensity of emotional experience: a social role interpretation. Journal of Personality and Social Psychology, 65(5), 1010-1022. https://doi. org/10.1037//0022-3514.65.5.1010

29. Hall, J. A. (1978). Gender effects in decoding nonverbal cues. Psychological Bulletin, 85(4), 845857. https://doi.org/10.1037/0033-2909.85.4.845

30. Hall, J. A. (1984). Nonverbal sex differences: Communication accuracy and expressive style. Johns Hopkins University Press.

31. Hall, J. A., \& Matsumoto, D. (2004). Gender differences in judgments of multiple emotions from facial expressions. Emotion, 4(2), 201-206. https://doi.org/10.1037/1528-3542.4.2.201

32. Hall, J. A., Carter, J. D., \& Horgan, T. G. (2000). Gender differences in nonverbal communication of emotion. In A. H. Fischer (Ed.), Studies in emotion and social interaction. Second series. Gender and emotion: Social psychological perspectives (p. 97-117). Cambridge University Press. https:// doi.org/10.1017/CBO9780511628191.006

33. Hampson, E., van Anders, S. M., \& Mullin, L. I. (2006). A female advantage in the recognition of emotional facial expressions: Test of an evolutionary hypothesis. Evolution and Human Behavior, 27(6), 401-416. https://doi.org/10.1016/j.evolhumbehav.2006.05.002

34. Hassin, R. R., Aviecer, H., \& Bentin, S. (2013). Inherently ambiguous: Facial expressions of emotions, in context. Emotion Review, 5(1), 60-65. https://doi.org/10.1177/1754073912451331

35. Herring, S. C., \& Dainas, A. R. (2020). Gender and age Influences on interpretation of emoji functions. ACM Transactions on Social Computing 3(2), 10. https://doi.org/doi.org/10.1145/3375629 
36. Hoffmann, H., Kessler, H., Eppel, T., Rukavina, S., \& Traue, H. C. (2010). Expression intensity, gender and facial emotion recognition: Women recognize only subtle facial emotions better than men. Acta Psychologica, 135(3), 278-283. https://doi.org/10.1016/j.actpsy.2010.07.012

37. Hyde, J. S. (2014). Gender similarities and differences. Annu. Rev. Psychol. 65, 373-398. https:// doi.org/10.1146/annurev-psych-010213-115057

38. Karama, S., Lecours, A. R., Leroux, J. M., Bourgouin, P., Beaudoin, G., Joubert, S., \& Beauregard, M. (2002). Areas of brain activation in men and men women during viewing of erotic film excerpts. Hum Brain Mapp, 16(1), 1-13. https://doi.org/10.1002/hbm.10014

39. Kinoshita, A., Okamoto, Y., Okada, G., Demoto, Y., Kunisato, Y., Yoshimura, S., Onoda, K., Kamachi, M., \& Yamawaki, S. (2012). Sex differences in neural activation to ambiguous facial expression in happy and sad context. Perceptual Motor Skills, 115(2), 349-59. https://doi.org/10.2466/22.24. PMS.115.5.349-359

40. Kret, M. E., \& De Gelder, B. (2012). A review on sex differences in processing emotional signals. Neuropsychologia, 50(7), 1211-1221. https://doi.org/10.1016/j.neuropsychologia.2011.12.022

41. Krys, K., Hansen, K., Xing, C., Domínguez, A., Szarota, P., \& Morales, M. F. (2015). It Is Better to Smile to Women: Gender Modifies Perception of Honesty of Smiling Individuals Across Cultures. International Journal of Psychology, 50(2), 150-154. https://doi.org/10.1002/ijop.12087

42. LaFrance, M., Hecht, M. A., \& Paluck, E. L. (2003). The contingent smile: A metaanalysis of sex differences in smiling. Psychological Bulletin, 129(2), 305-334. https://doi.org/10.1037/00332909.129.2.305

43. Lee, T., Liu, H. L., Hoosaine, R., Liaod, W-T., Wud, C-T., Yuena, K., Chanf, C., Foxg, P. T., \& Gaog, J. H. (2002). Gender differences in neural correlates of recognition of happy and sad faces in humans assessed by functional magnetic resonance imaging. Neuroscience Letters 333, 13-16. https://doi.org/10.1016/S0304-3940(02)00965-5

44. Lyusin, D., \& Ovsyannikova, V. (2016). Measuring two aspects of emotion recognition ability: Accuracy vs. sensitivity. Learning and Individual Differences, 52, 129-136. https://doi.org/10.1016/j. lindif.2015.04.010

45. Matsumoto, D., \& Hwang, H (2010). Judging faces in context. Social and Personality Psychology Compass, 4(6), 393-402. https://doi.org/10.1111/j.17519004.2010.00271.x

46. Mazurski, E. J., Bond, N. W., Siddle, D. A., \& Lovibond, P. F. (1996). Conditioning with facial expressions of emotion: Effects of CS sex and age. Psychophysiology, 33(4),416-425. https://doi. org/10.1111/j.1469-8986.1996.tb01067.x

47. McClure, E. B. (2000). A meta-analytic review of sex differences in facial expression processing and their development in infants, children, and adolescents. Psychological Bulletin, 126(3), 424453. https://doi.org/10.1037/0033-2909.126.3.424

48. Montagne, B., \& Kessels, R. P. (2005). Frigerio E, de Haan EH, \& Perrett DI. Sex differences in the perception of affective facial expressions: do men really lack emotional sensitivity? Cognitive Processing, 6(2), 136-141. https://doi.org/10.1007/s10339-005-0050-6

49. Niedenthal, P. M., Krauth-Gruber, S., \& Ric, F. (2006). Psychology of emotion: Interpersonal, experiential and cognitive approaches. Psychology Press.

50. Prada, M., Rodrigues, D. L., Garrido, M. V., Lopes, D., Cavalheiro, B., \& Gasparb, R. (2018). Motives, frequency and attitudes toward emoji and emoticon use. Telematics and Informatics, 35(7), 1925-1934. https://doi.org/10.1016/j.tele.2018.06.005

51. Rahman, Q., Wilson, G. D., \& Abrahams, S. (2004). Sex, sexual orientation, and identification of positive and negative facial affect. Brain and Cognition, 54(3), 179-185. https://doi.org/10.1016/j. bandc.2004.01.002

52. Sawada, R., Sato, W., Kochiyama, T., Uono, S., Kubota, Y., Yoshimura, S., \& Toichi, M. (2014). Sex differences in the rapid detection of emotional facial expressions. PloS One, 9(4), e94747. https://doi.org/10.1371/journal.pone.0094747

53. Saylik, R., Raman, E., \& Szameitat, A. J. (2018). Sex differences in emotion recognition and working memory tasks. Frontiers in Psychology, 9, 1072. https://doi.org/10.3389/fpsyg.2018.01072

54. Schutte, N. S., Malouff, J. M., Hall, L. E., Haggerty, D. J., Cooper, J. T., Golden, C. J., \& Dornheim, L. (1998). Development and validation of a measure of emotional intelligence. Personality and individual differences, 25(2), 167-177. https://doi.org/10.1016/S0191-8869(98)00001-4

55. Shields S. A. (2002). Speaking from the heart: Gender and the social meaning of emotion. Cambridge University Press.

56. Spence, J. T., \& Helmreich, R. L. (1978). Masculinity and femininity: Their psychological dimensions, correlates, and antecedents. University of Texas Press. 
57. Thompson, A. E., \& Voyer, D. (2014). Sex differences in the ability to recognise non-verbal displays of emotion: A meta-analysis. Cognition and Emotion, 28(7), 1164-1195. https://doi.org/10.1 $\underline{080 / 02699931.2013 .875889}$

58. Tottenham, N., Tanaka, J.W., Leon, A.C., McCarry, T., Nurse, M., Hare, T.A., Marcus, D.J., Westerlund, A., Casey, B.J., \& Nelson, C. (2009). The NimStim set of facial expressions: judgments from untrained research participants. Psychiatry Research, 168, 242-249. https://doi.org/10.1016/j. psychres.2008.05.006

59. Van Kleef, G. A., \& De Dreu, C. K. W. (2010). Longer-term consequences of anger expression in negotiation: Retaliation or spillover? Journal of Experimental Psychology, 46(5), 753-760. https:// doi.org/10.1016/j.jesp.2010.03.013

60. Vrana, S. R., \& Rollock, D. (2002). The role of ethnicity, gender, emotional content, and contextual differences in physiological, expressive, and self-reported emotional responses to imagery. Cognition \& Emotion, 16(1), 165-192. https://doi.org/10.1080/02699930143000185

61. Wieser, M. J., \& Brosch, T. (2012). Faces in context: a review and systematization of contextual influences on affective face processing. Frontiers in Psychology, 3, 471. https://doi.org/10.3389/ fpsyg.2012.00471

62. Wild, B., Erb, M., \& Bartels, M. (2001). Are emotions contagious? Evoked emotions while viewing emotionally expressive faces: quality, quantity, time course and gender differences. Psychiatry Research, 102(2), 109-24. https://doi.org/10.1016/s0165-1781(01)00225-6

63. Wingenbach, T. S. H., Ashwin, C., \& Brosnan, M. (2018). Sex differences in facial emotion recognition across varying expression intensity levels from videos. PLoS ONE, 13(1), e0190634. https://doi.org/10.1371/journal.pone.0190634

64. Yoon, K. L., Joormann, J., \& Gotlib, I. H. (2009). Judging the Intensity of Facial Expressions of Emotion: Depression-Related Biases in the Processing of Positive Affect. Journal of Abnormal Psychology, 118(1), 223-228. https://doi.org/10.1037/a0014658

RECIBIDO: 08 de septiempre de 2020 MODIFICADO: 20 de mayo de 2021 ACEPTADO: 24 de mayo de 2021 